\title{
Radar Images of Penetrable Targets Generated from Ramp Profile Functions
}

\author{
Soumya Nag, Member, IEEE, and Leon Peters, Jr., Life Fellow, IEEE
}

\begin{abstract}
Images can be generated for penetrable targets from their scattered fields when the time dependence of the incident electromagnetic (EM) wave takes the form of a ramp function. Previous researchers have developed these concepts for conducting targets. This paper focusses attention on penetrable targets. Ramp response signatures of the targets for cases where the dielectric constant of the target is greater than and also less than that of the ambient medium are included. The latter case can be applied as a signature of antipersonnel mines. The results contained herein are based on: 1) scattering measurements in The Ohio State University ElectroScience Laboratory Compact Range; 2) scattering computation using an eigenfunction solution and a method of moments solution; and 3) a very limited set of measurements generated from a buried land mine using the ElectroScience Laboratory Ground Penetrating Radar. The targets presented in this paper include metallic and dielectric spheres and actual land mines.
\end{abstract}

Index Terms-Dielectric scatterers, ground-penetrating radar (GPR), land mines.

\section{INTRODUCTION}

$\mathbf{T}$ HE ramp response of a radar target is defined herein as the far zone backscattered time-domain waveform resulting from a plane wave illumination whose time dependence is that of a ramp function. This ramp incident wave is a function of time and distance. The backscattered field from a ramp incident wave is proportional to the transverse cross-sectional area corresponding to the time and position on the target such that the argument of the incident ramp function is zero. This property has been demonstrated for conducting targets by use of physical optics (PO) and has been used to create images of such targets from only three orthogonal incidence angles. These concepts have been recently expanded to treat dielectric bodies.

Consequently, it represents a potential valuable identification tool for buried mines. It has several advantages over other ground-penetrating radar (GPR) incident waves. It is basically a low-frequency concept, and consequently the clutter for a typical case is decreased. In most earth media, the conductivity and the attenuation factor are increasing functions of frequency over GPR frequency bands. The most distinguishable characteristic of a mine as well as similar false targets is observed for frequen-

Manuscript received September 1, 1999; revised May 3, 2000. This work was supported by Duke University under an award from ARO (OSD MURI Program).

S. Nag is with Time Domain Corporation, Huntsville, AL 35806 USA (e-mail: soumya.nag@ timedomain.com).

L. Peters, Jr., is with the ElectroScience Laboratory, Department of Electrical Engineering, The Ohio State University, Columbus, OH 43212 USA (e-mail: peters.6@osu.edu).

Publisher Item Identifier S 0018-926X(01)02290-6. cies just above the Rayleigh region, thus enhancing identification capabilities.

Applications of electromagnetic (EM) field waveforms, produced by scattering of transient plane waves from finite objects, to target identification was first discussed by Kennaugh and Moffatt [1]. Using physical optics, they showed that the scattered field from a finite metallic target due to a ramp driving function traces out the cross-sectional area profile of the target as a function of a distance along the line-of-sight (known as the profile function of the target). Young [2], [3] compared the measured ramp response signatures for different metallic targets of simple shapes with the corresponding profile functions. Using the ramp waveforms, he also generated three-dimensional images of simple conducting targets in free-space from orthogonal look angle radar data.

Nag et al. [4] have adapted the original solution to include dielectric (or penetrable) targets and have obtained the ramp profiles and images for such targets.

The ramp response of a target has the following characteristics. It is derived from the specular or early time component of the target backscattered impulse response. The ramp response is a low-frequency concept, since it is obtained by taking the inverse Fourier transform (IFT) of the target backscattered frequency response spectrum weighted by the inverse of the frequency squared. Other types of imaging techniques require target radar echo measurements at multiple antenna positions to form a usable image of the target. However, generation of an image of a target of simple shape via ramp waveforms requires the ramp response obtained typically from three antenna positions.

The ramp response has some inherent limitations. First, since the ramp response is a low-frequency phenomenon, it is essential for the lowest of the interrogating frequency components to lie in the Rayleigh region of the target's backscattered radar echo so that a valid ramp waveform can be generated. Since plane wave incidence is used, a large antenna could be required. The multiple reflections of waves inside a inhomogeneous penetrable target can perturb the ramp waveform from the desired transverse cross-sectional area of the target along line-of-sight. There are signal-processing techniques available to reduce such multiple reflections. Three-dimensional images of dielectric targets in free-space are presented using the processed ramp waveform obtained from three orthogonal look angles. For GPR applications, means of generating images of buried targets are also suggested.

The organization of this paper is as follows. In Section II, we discuss the theory of the ramp response for dielectric (penetrable) bodies. In Section III, we present the ramp response 


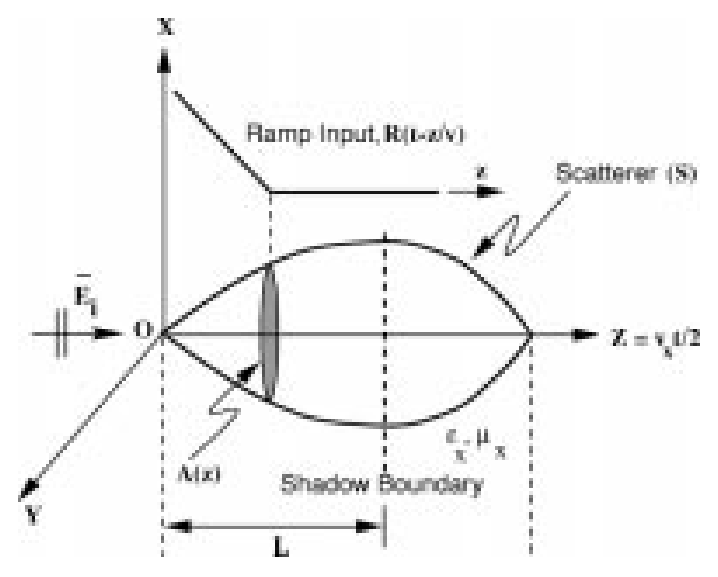

Fig. 1. Target geometry and ramp incident wave.

signatures of dielectric and conducting bodies immersed in both free-space and more dense dielectric media. In Section IV, complete three dimensional (3-D) images of land mines, obtained using the ramp response for three orthogonal look angles, are presented. Section V discusses the potential measurement of ramp response of buried targets using a dielectric rod antenna, developed at The Ohio State University ElectroScience Laboratory (OSU-ESL) [5]. In Section VI, an image of a buried land mine is shown, which is obtained from the measured GPR data. Finally, in Section VII, some concluding remarks are presented.

\section{RAmP ResPonse FOR PENETRABle TARgets}

The ramp incident wave $R(z, t)$ is a time-spatial function given by

$$
R(z, t)=R\left(t-\frac{z}{v_{x}}\right)
$$

where $t$ is time and $v_{x}$ is the velocity of the wave in the ambient medium. The target geometry, as a function of coordinate $z$, is illustrated in Fig. 1 for a given time instant. It has been shown in [2] and [3] that the ramp response for a conducting target is given by

$$
f_{r}^{M}(t)=-\frac{1}{\pi v_{x}^{2}} A\left(\frac{v_{x} t}{2}\right)
$$

where $A\left(v_{x} t / 2\right)$ is the transverse cross-sectional area of the target and the argument $\left(v_{x} t / 2\right)$ accounts for the two-way radar path $2 z=v_{x} t$.

The ramp response given in (2) for conducting targets is extended to dielectric targets by modifying the reflection coefficients that are used in the PO solution [1], [6]. In this case, the reflection coefficients become distance (or time) dependent because of their angle of incidence dependence for dielectric media. The ramp response for penetrable targets via PO takes the form

$$
f_{r}^{D}(t)=\frac{1}{\pi v_{x}^{2}} \int_{0^{-}}^{t} \bar{R}_{12}\left(\frac{v_{x} t^{\prime}}{2}\right) \frac{d}{d t^{\prime}} A\left(\frac{v_{x} t^{\prime}}{2}\right) d t^{\prime}
$$

where $\bar{R}_{12}\left(v_{x} t^{\prime} / 2\right)$ is the average reflection of the dielectric surface. For a rotationally symmetric target

$$
\bar{R}_{12}=\frac{1}{2}\left[R_{12}^{\perp}+R_{12}^{\|}\right]
$$

where $R_{12}^{\perp}$ and $R_{12}^{\|}$are the Fresnel reflection coefficients of a dielectric surface perpendicular and parallel to the plane of incidence, respectively. Because of the functional form of $R_{12}^{\perp}, R_{12}^{\|}, \bar{R}_{12}$ can be approximated by their values at normal incidence. Then $\bar{R}_{12}(0)$ can be taken outside the integral of (3), and we obtain

$$
f_{r}^{D}(t)=\frac{\bar{R}_{12}(0)}{\pi v_{x}^{2}} A\left(\frac{v_{x} t}{2}\right) .
$$

$A\left(v_{x} t / 2\right)$ is maximum at the shadow boundary of the target. Also, the duration of the ramp function from zero to its maximum value is approximately

$$
t_{d}=L \sqrt{\frac{\epsilon_{r}^{\mathrm{rel}}}{15.24}} \mathrm{~ns}
$$

where $L$ is the distance from the onset of the target to the shadow boundary, as shown in Fig. 1 in centimeters, and $\epsilon_{r}^{\text {rel }}$ is the relative permittivity of the external medium. While this is valid over the lit region, it has also been found to represent a useful approximation in the shadow region when $L$ is replaced by the length along the surface of the target from the shadow boundary to the furthest point on the target.

The ramp response of a scatterer can be obtained from its backscattered frequency response in two ways.

1) By double integrating the impulse response of the scatterer, i.e.,

$$
f_{r}(t)=\int_{t^{\prime}=0^{-}}^{t} \int_{\tau=0^{-}}^{t^{\prime}} f_{I}(\tau) d \tau d t^{\prime} .
$$

The impulse response $f_{I}(t)$ is obtained via IFT of the backscattered frequency response.

2) By performing IFT of a target's backscattered frequency response $F_{I}(j \omega)$ weighted by $1 /(j \omega)^{2}$, i.e.,

$$
f_{r}(t)=\operatorname{IFT}\left[F_{R}(j \omega)\right]=\operatorname{IFT}\left[\frac{1}{(j \omega)^{2}} F_{I}(j \omega)\right] .
$$

Note that (7) would also contain the additive terms of the form $c_{0} t+c_{1}$, where $c_{0}$ and $c_{1}$ are constants. $c_{0}$ and $c_{1}$ are the dc biases of the step and ramp responses, respectively. $c_{0}$ is simply subtracted from the step response (single integral of the impulse response) before it is integrated once, and $c_{1}$ can be subtracted from the ramp response. Also in $(8), F_{R}(j \omega)$ contains additional additive terms: $\left(1 /(j \omega)^{2}\right) \int_{-\infty}^{0-} f_{i}(\tau) d \tau+(1 / j \omega) \int_{-\infty}^{0-}\left\{\int_{-\infty}^{\tau} f_{i}\left(t^{\prime}\right) d t^{\prime}\right\} d \tau$. But these terms drop out since we are interested in zero initial conditions, i.e., $\int_{-\infty}^{0-} f_{i}(\tau) d \tau=0$ and $\int_{-\infty}^{0-} f_{u}(\tau) d \tau=0$, where $f_{u}(t)$ is the step response.

Fig. 2 is a plot of the physical optics ramp responses, as obtained from (2), for a perfectly conducting sphere of $7.6 \mathrm{~cm}$ diameter. Also shown in Fig. 2 is the result obtained using (3) 


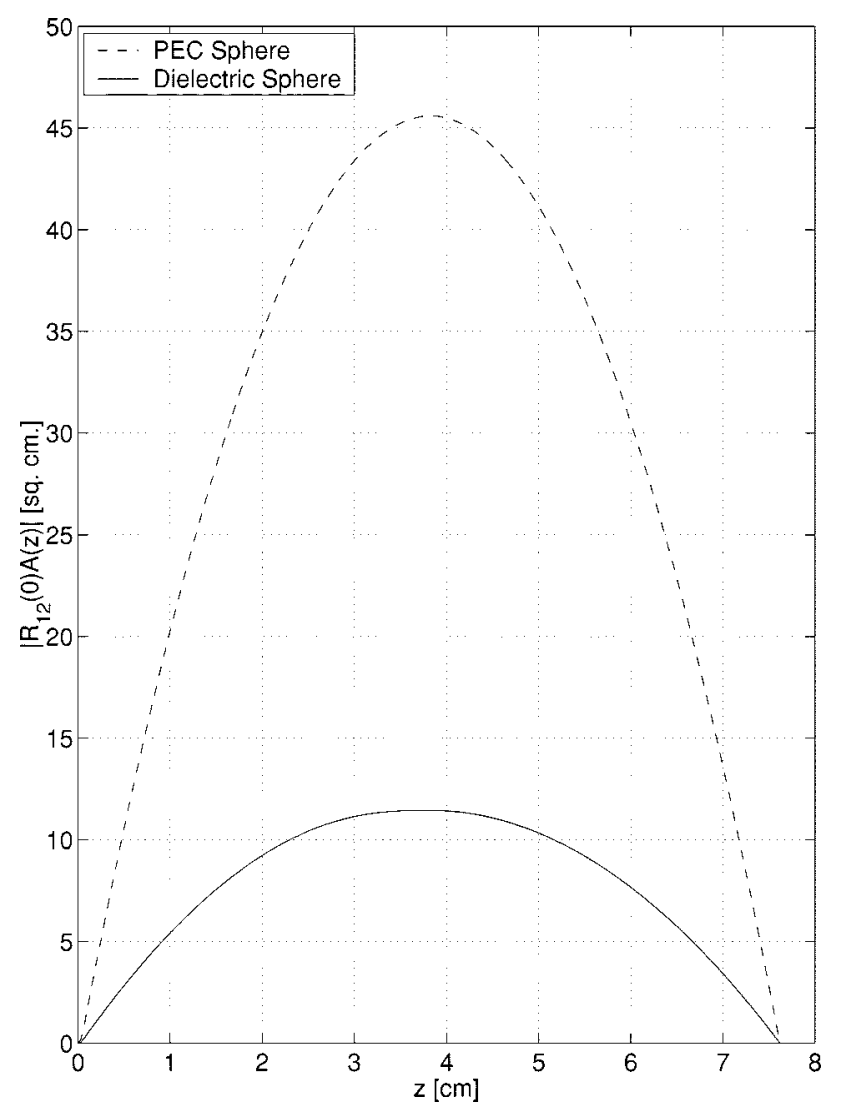

Fig. 2. PO ramp response functions of $7.6 \mathrm{~cm}$ diameter conducting and of dielectric spheres $\left(\epsilon_{i}=3\right)$ in free-space.

for a dielectric sphere $\left(\epsilon_{i}^{\mathrm{rel}}=3\right)$ of the same size. The difference in the amplitude of $f_{r}(t)$ for the two spheres is due to the different values of the reflection coefficient. In this case, the function $(1 / 2)\left(R^{\perp}+R^{\|}\right) \approx R^{\perp}(0)=R^{\|}(0)$. Note that there would be only small differences between these two cases if the curve for the dielectric sphere had been normalized to that of the conducting sphere. For this case, variations of $R^{\perp}\left(v_{x} t / 2\right)$ and $R^{\|}\left(v_{x} t / 2\right)$ tend to cancel each other. This result, of course, is valid for symmetric geometries. However, if the dielectric target is a cylindrical body, at endfire incidence the reflection coefficient must be retained inside the integral of (3).

\section{RAMP PROFILE FunCTION}

The interest in the ramp response of targets is dictated by its close relationship to the physical geometry of the target. This is illustrated in Fig. 3, in which the transverse cross-sectional area obtained from the ramp response (also referred to in the paper as the ramp profile function) of both a conducting sphere and a dielectric $\left(\epsilon_{i}^{\text {rel }}=3\right)$ in free-space, are shown. The profile function is simply its physical transverse cross sectional area as a function of range ( $z$ of Fig. 1). The ramp response is obtained from the exact solution of the scattered fields of each sphere for $0.02-8.02 \mathrm{GHz}$ frequency band. The agreement between the profile function and the ramp response in the lit region is excellent. The normalized impulse response, obtained from the exact solution, for the dielectric sphere is also shown, and it is much more complicated than the ramp response.

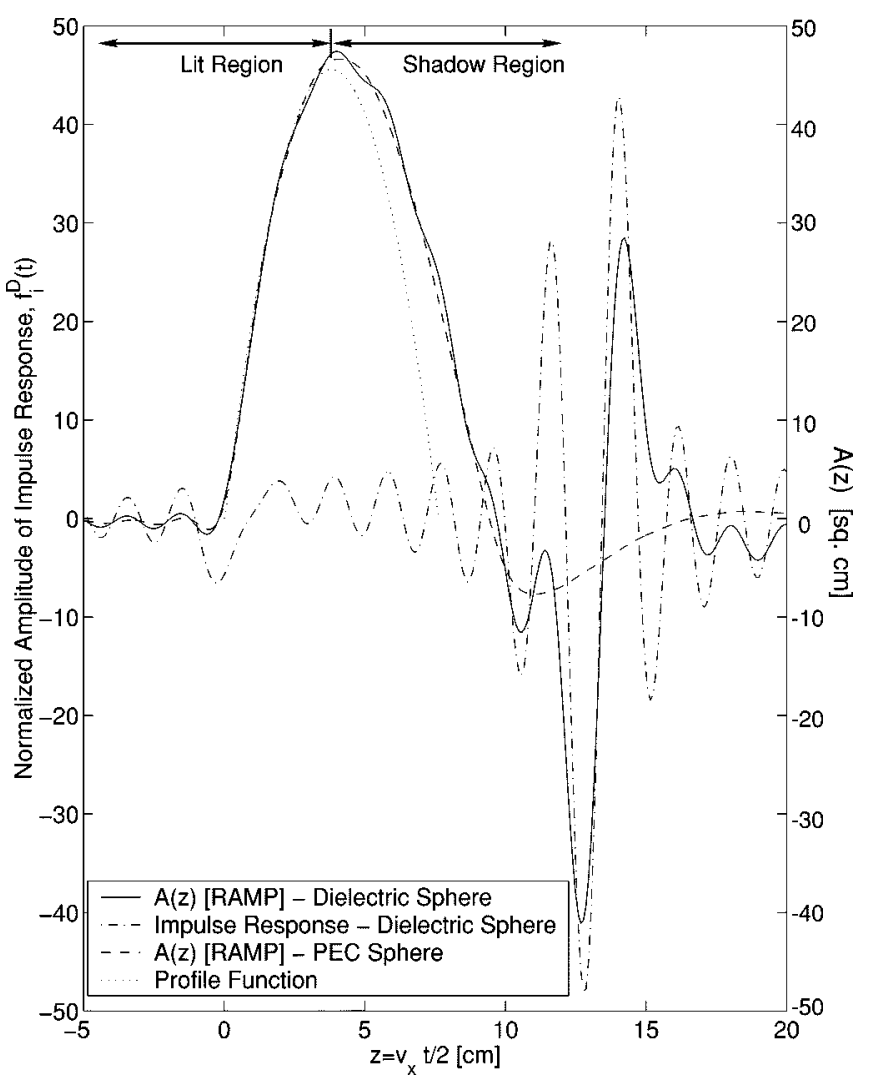

Fig. 3. Normalized impulse response and the transverse cross-sectional area $A(z)$, obtained from the ramp response, for a 7.6-cm diameter PEC sphere and dielectric $\left(\epsilon_{i}^{\mathrm{rcl}}=3\right)$ sphere in free-space.

If the operational frequency band does not contain sufficiently low-frequency components, the ramp waveform contains an unwanted low-frequency ripple corresponding to the minimum frequency $\left(f_{\min }\right)$ used in the Rayleigh region. These sidelobes can be reduced by simply extrapolating the magnitude and phase of the $1 /(j \omega)^{2}$ weighted frequency response to $f_{\min } \rightarrow 0$, respectively, as independent of frequency and linear function of frequency. Note that in the Rayleigh region of the backscattered frequency response, the magnitude is approximately proportional to $\omega^{2}$ and the phase is a linear function of $\omega$. However, this extrapolation is valid only if the minimum radar frequency lies in the Rayleigh region of the target in a given medium.

Ramp waveforms for a number of targets have been obtained from scattered field data for dielectric targets in free-space, dielectric targets in penetrable medium, and dielectric targets immersed in a lossy dispersive medium [7]. Additional processing is required for these latter cases. When the dielectric constant of the medium in greater than that of the target, internal reflections occur at times when the ramp is being generated, and these can be removed from the impulse response before forming the ramp response. This will also be the case for mines whose internal structure is more complex. However, even if these reflections are not removed, the ramp response still provides usable data. A more complex solution is required if the medium is lossy and dispersive. A means of treating this case is discussed in a recent paper [8]. 


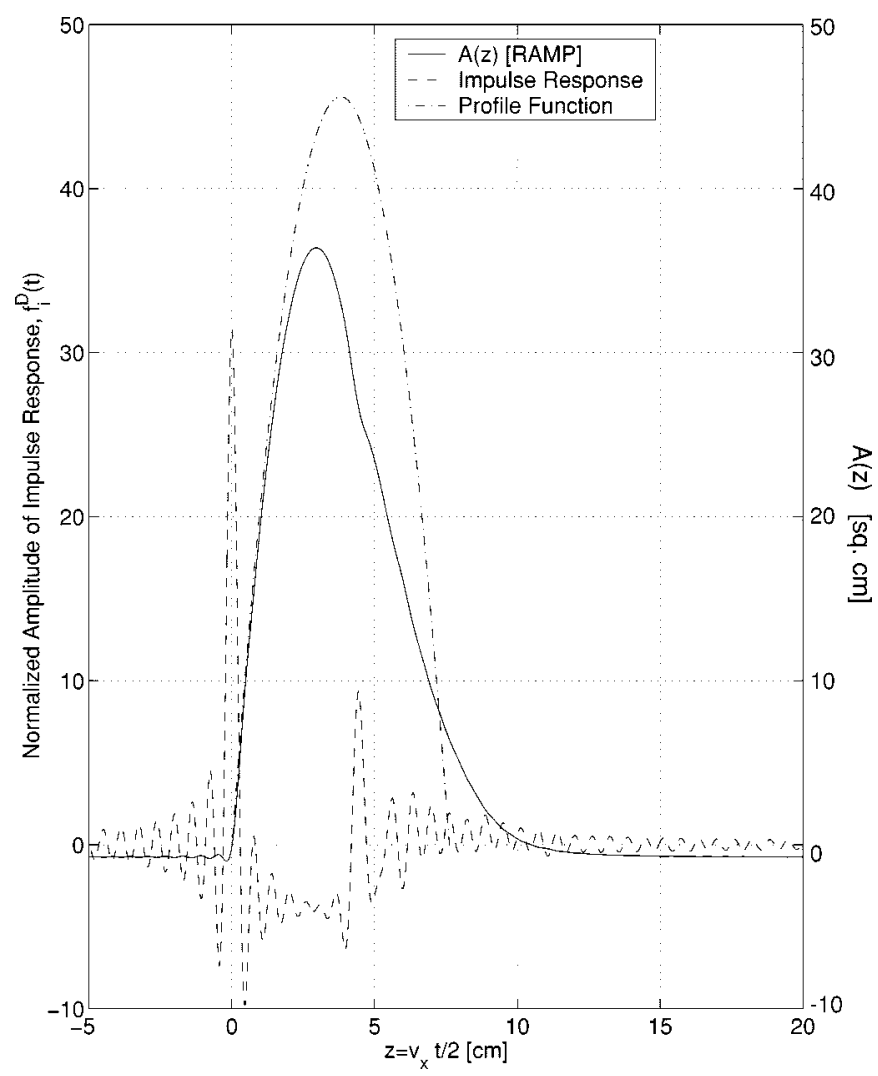

Fig. 4. Impulse and ramp responses of a 7.6-cm diameter lossless dielectric sphere with $\epsilon_{i}^{\mathrm{rel}}=3$ embedded in lossless dielectric medium of $\epsilon_{x}^{\mathrm{rcl}}=9$.

The results obtained using an eigenfunction solution for a dielectric sphere $\left(\epsilon_{i}^{\mathrm{rel}}=3\right)$ immersed in a dielectric medium $\left(\epsilon_{x}^{\text {rel }}=9\right)$ are shown in Fig. 4. There are some mechanisms that can distort the ramp profile functions. The impulse response of Fig. 4 contains a number of reflections that can cause perturbations of the ramp response. These can be reduced to improve the shape of the ramp response using signal processing developed to remove the ground-free-space interface reflections [9]. However, the major perturbation appears to occur when the angle of incidence approaches the critical angle, and the approximation implicit in (5) is no longer valid. Further analysis of this case is beyond the scope of this paper. While the ramp response for this case is disturbed, it can be used to provide an image whose radius would be slightly smaller than the actual radius of the original sphere.

The ramp response for normal incidence on a 2.54-cm dielectric cube measured in free-space represents an extreme case. It would require a very large bandwidth to obtain the sharp rise time expected for such a cube. Consequently, the frequency band was increased from 6 to $12 \mathrm{GHz}$ for the cube [7]. In this case, the ramp waveform is slightly delayed about $0.51 \mathrm{~cm}$ from the profile function but otherwise gives good agreement for the greater bandwidth.

The ramp response of a pair of dielectric blocks $\left(\epsilon_{i}^{\text {rel }}=3\right)$, immersed in a dielectric medium $\left(\epsilon_{x}^{\text {rel }}=9\right)$ where $\epsilon_{x}^{\text {rel }}>\epsilon_{i}^{\text {rel }}$, has also been reported previously. Ramp responses for a dielectric block attached to a larger one have also been generated using a method of moment (MoM) solution [10]. Both blocks are clearly recognizable when the small block is directly illu- minated. The presence of the smaller block is detectable, but its response is distorted when it is in the shadow of the larger block [7].

Measured free-space scattered fields $(0.1-6.0 \mathrm{GHz}$ bandwidth) are used to generate the ramp response for an SB-33 anti-personnel (AP) land mine for broadside and endfire incidence, shown in Fig. 5(a) and (b). The broadside and endfire dimensions for this mine are 8.8 and $3.2 \mathrm{~cm}$, respectively. Note that the duration of the ramp response is not seriously perturbed by the internal reflections.

Finally, ramp response, generated using computed scattered fields, have been presented elsewhere [5] for a perfectly conducting sphere and cube immersed in a lossy dispersive medium $\left(\epsilon_{x}^{\mathrm{rel}}=9, \sigma_{x}=0.8 \mathrm{~S} / \mathrm{m}\right)$. An eigenfunction solution for $0.3-3.1$ $\mathrm{GHz}$ frequency band, and an MoM solution for $0.3-4.3 \mathrm{GHz}$ frequency band, were used to generate the backscattered fields for the sphere and the cube, respectively. In the lower part of the frequency band of interest, the medium is a conductor; in the upper part, it is a lossy dielectric; and in intermediate region, it is neither. It is emphasized that this medium would be far too lossy for any ground. Nevertheless, after substantial additional processing, the ramp responses in this lossy dispersive medium approximate the profile function particularly in the lit region of the target [8].

\section{IMAges of Dielectric Bodies IN FreE-Space ObTAined FROM THE RAMP RESPONSE}

A substantial amount of information is generated from a single ramp response of a target concerning its length, width, and electrical properties. Much more can be generated in the form of images created by combining results from different incidence angles. Young [2], [3] developed this concept using three orthogonal observation angles, and this approach is used to obtain some of the images to be presented. The reader is referred to the above references for the details of this imaging process. Young considered only metallic targets where the reflection coefficient is independent of incidence angle. For dielectric targets, the reflection coefficient is as yet an unknown variable depending on the incidence angle and the electrical properties of the ambient medium. Hence, it becomes difficult to evaluate $A(x), A(y)$, and $A(z)$ from the measured ramp waveforms. Images of several land mines have been generated, and two of these are shown in in Figs. 6 and 7, which were obtained using Young's imaging technique and three orthogonal incidence angle ramp responses for the $0.1-6.0 \mathrm{GHz}$ bandwidth. The duration and the shape of the three orthogonal ramp waveforms have been used to normalize the peak of each of these waveforms to the maximum value of the transverse cross-sectional $\left(A_{\max }\right)$ area in each orthogonal direction. For targets with a flat surface, the target width obtained in the image can be too large. This is because the rise (and fall) times of the ramp waveforms for the front surfaces of these targets are stretched due to the limited bandwidth. This problem can be alleviated by extending the bandwidth. Otherwise, the images are reasonable pictures of the respective targets.

Most mines have rotational symmetry. If such mines, buried in the ground, are oriented with the axis of rotation almost 


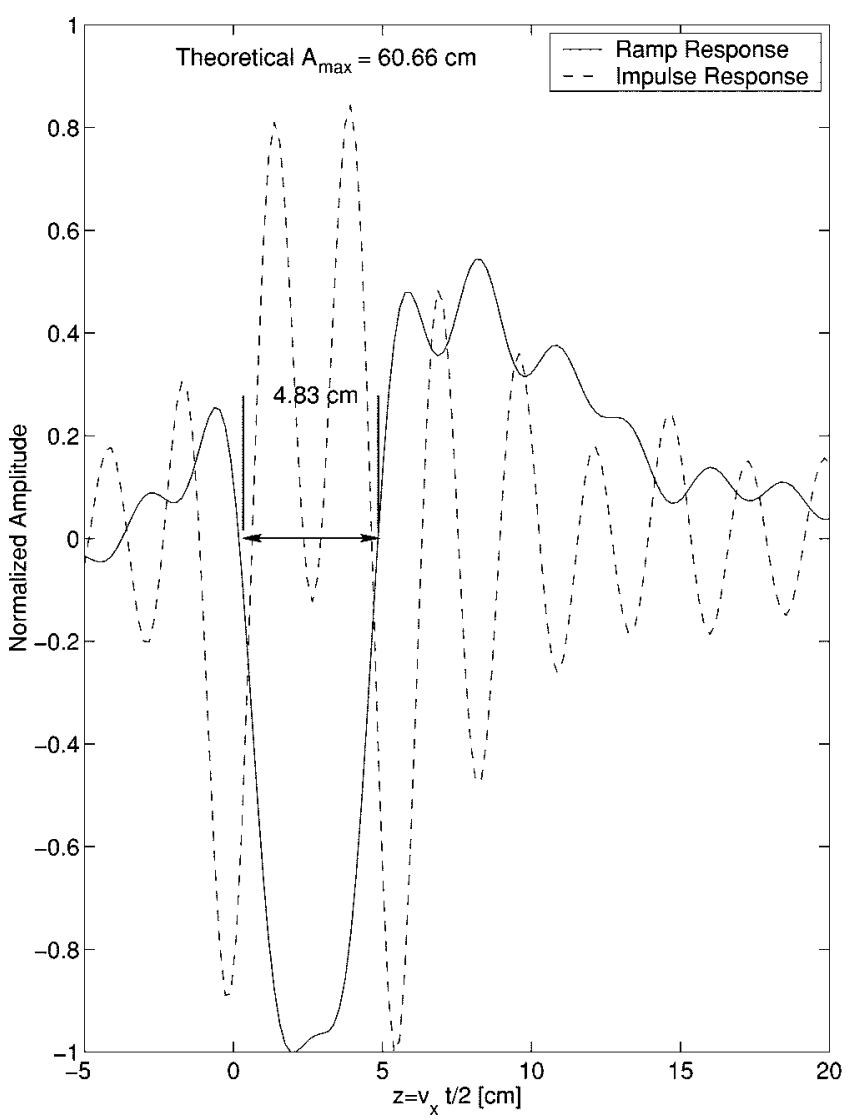

(a)

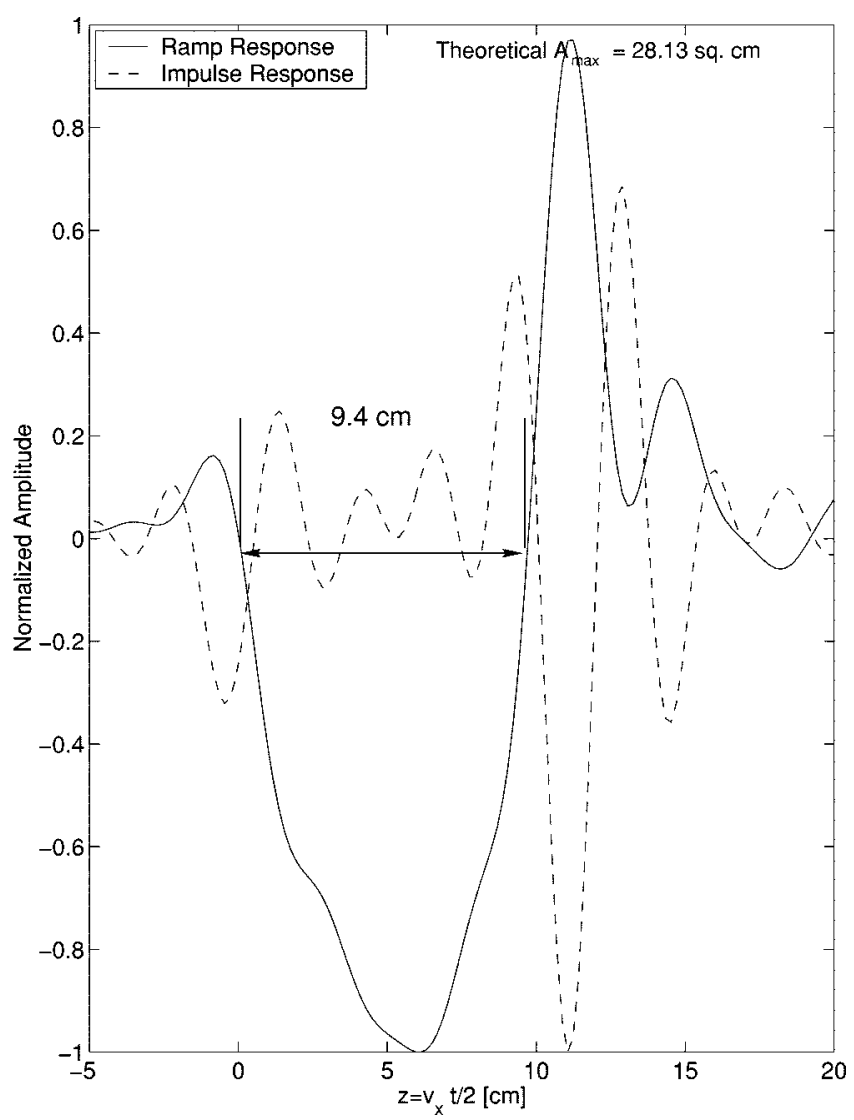

(b)

Fig. 5. Normalized ramp response and impulse response for the land mine SB-33 measured at OSU-ESL Compact range for (a) broadside and (b) endfire incidence.

perpendicular to the ground-air interface, only a single ramp response is required to generate an image. The rotational symmetry of a buried target can be demonstrated by measuring the target response as a function of antenna positions from different directions. The transverse cross-sectional function $A(z)=\pi r^{2}(z)$, where the radius $r(z)$ completely approximates the geometry. The maximum value (peak of the ramp profile function) $A_{\max }$ for a buried target could be generated in two ways: 1) by using the usual GPR measured data as the antenna is moved over the mine to estimate its lateral extent and 2) from (4) by using the maximum measured value of the ramp profile function, which implies that accurate, calibrated data is available. This last method is used to generate an image of an SB-33 mine in free-space using only the ramp waveform, given in Fig. 5(a), and assuming the rotational symmetry of the body. The resulting image is shown in Fig. 8 .

\section{Potential Measurement of Ramp Response Using GPR WITH A DIELECTRIC ROD ANTENNA}

In the preceding discussion, results have been generated under ideal conditions either from measured data taken in the OSU-ESL compact range or computed data. The task now is to generate these data using a GPR capable of acquiring the requisite data under field conditions. As has been observed, the fields of the focused reflector [11] do approximate a plane wave in the focused spot region, but this antenna would be far

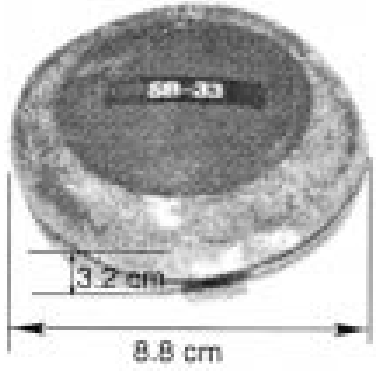

(a)

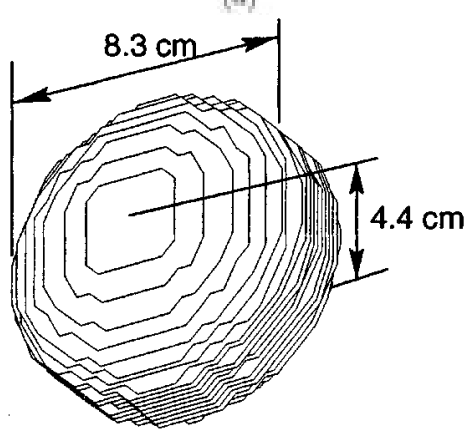

(b)

Fig. 6. (a) A-P mine SB-33 in free-space and (b) reconstructed image of the land mine.

too large to include the required scattered fields in the Rayleigh region of A-P mines. 


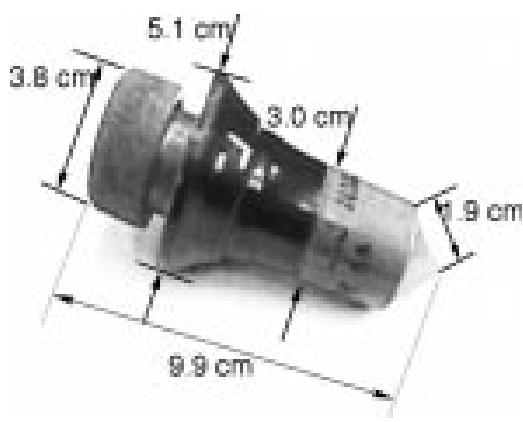

(a)

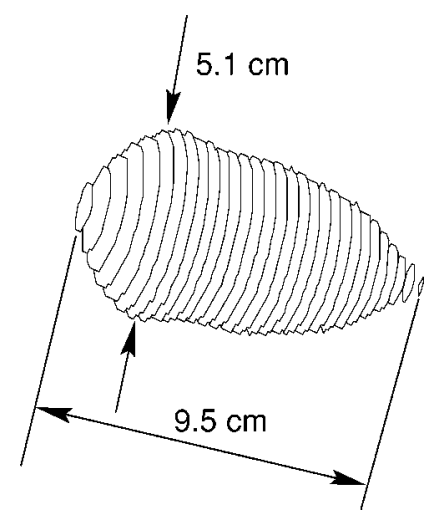

(b)

Fig. 7. (a) A-P mine C4A1 in free-space, and (b) reconstructed image of the mine.

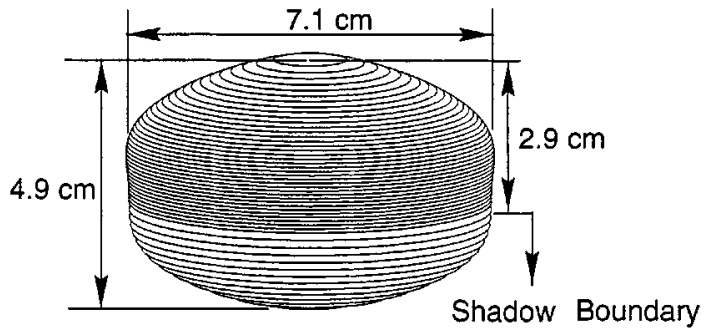

Fig. 8. An image of SB-33 measured in free-space, using only the ramp response given in Fig. 5.

The second type of antenna used in GPR for mine detection is a wide-band dielectric rod antenna (near-field probe) that also creates a highly localized illumination [5]. This dielectric rod antenna, however, could be hand held or vehicle-mounted for convenience and speed. It is interesting to note that this antenna is in the far field of the mine at a range of $7.6 \mathrm{~cm}$ up to $2 \mathrm{GHz}$. This is obtained using the range $R>\left(2 D^{2} / \lambda\right)$ criterion and assuming $D=7.6 \mathrm{~cm}$ for a typical A-P mine. If the range is increased to $22.8 \mathrm{~cm}$, the frequency increases to $6 \mathrm{GHz}$. This does not even include the refraction at the ground-air interface, which would further reduce this phase error.

It is suggested that plane wave representation could be improved by taking a series of measurements as the antenna is moved over the target. These time-domain waveforms could simply be averaged to further reduce the phase error inherent in the dielectric rod antenna pattern, which is simply a point source radiator. A radar can be used even though certain mechanisms such as the creeping wave on a sphere would not be observed.

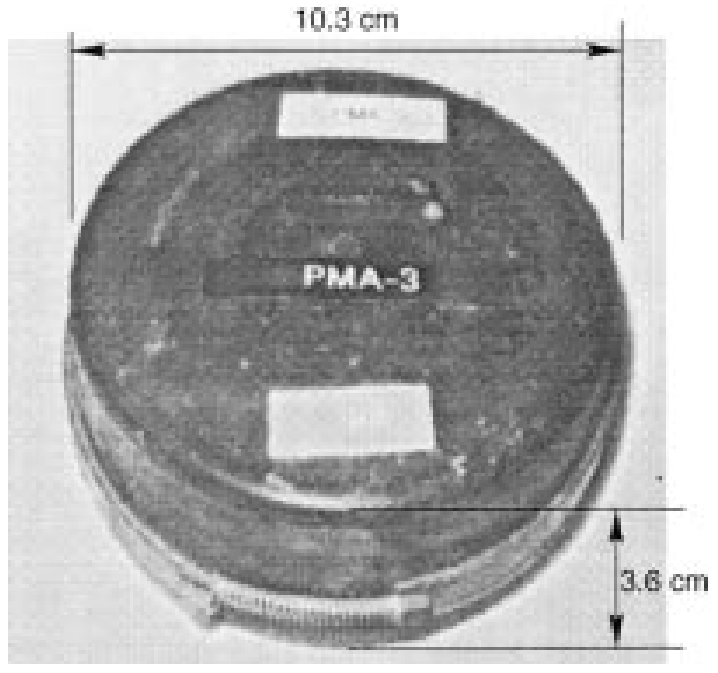

Fig. 9. PMA-3 A-P mine.

However, these mechanisms would only corrupt the data to be used in generating these ramp images.

The current dielectric rod antenna operates from 1 to $6 \mathrm{GHz}$ and does not include the Rayleigh region of most of the A-P mines buried in clay. A simple redesign should produce such an antenna that operates as low as $300 \mathrm{MHz}$ up to the Rayleigh region.

A more serious problem is that of launching a wave parallel to the ground-air interface to achieve the required orthogonal incidence on the target. The antenna would be another version of the dielectric rod antenna. This antenna would have a dielectric constant $\epsilon_{a}$ greater than that of the earth $\epsilon_{x}$. The antenna would require contact with the ground and would be at an angle $\theta$ greater than the critical angle measured from the normal to the ground, or $\sin \theta>\sqrt{\epsilon_{x} / \epsilon_{a}}$, so that the lateral wave is launched below the ground.

\section{RAMP IMAGE OF A MiNE BURIED IN DRY ClAY}

The goal of this section is to demonstrate that a measured ramp response scan be obtained for an actual mine in a real medium with clutter present. It further demonstrates that a GPR using the dielectric rod antenna can be calibrated with sufficient accuracy to generate a realistic image. The PMA-3 A-P mine (see Fig. 9) buried approximately $2.5 \mathrm{~cm}$ deep in a dry clay medium at a JUXOCO site, Fort A.P. Hill, Virginia, was selected for this purpose. A network analyzer and dielectric rod was used as the GPR. The measured strength of the impulse response is shown in Fig. 10 as a function of time (ns) and antenna position $(\mathrm{cm})$. The stripes across the top of this figure (at around $0.2 \mathrm{~ns}$ ) are the response due to the ground reflections. They can be removed from the data using a signal-processing scheme developed by van der Merwe [12]. The scattering from the mines occurs at about $-0.54 \mathrm{~ns}$ to $-1.35 \mathrm{~ns}$ and 7.6-20.3 $\mathrm{cm}$ antenna position range, as indicated by the hyperbolic arc in the figure. This time-domain plot is obtained via IFT on 1.2-6 $\mathrm{GHz}$ range frequency-domain response. The measured data at $12.7-\mathrm{cm}$ position is time gated between the -0.54 to $-1.35 \mathrm{~ns}$ range and is then transformed to the frequency domain (1.2-6 

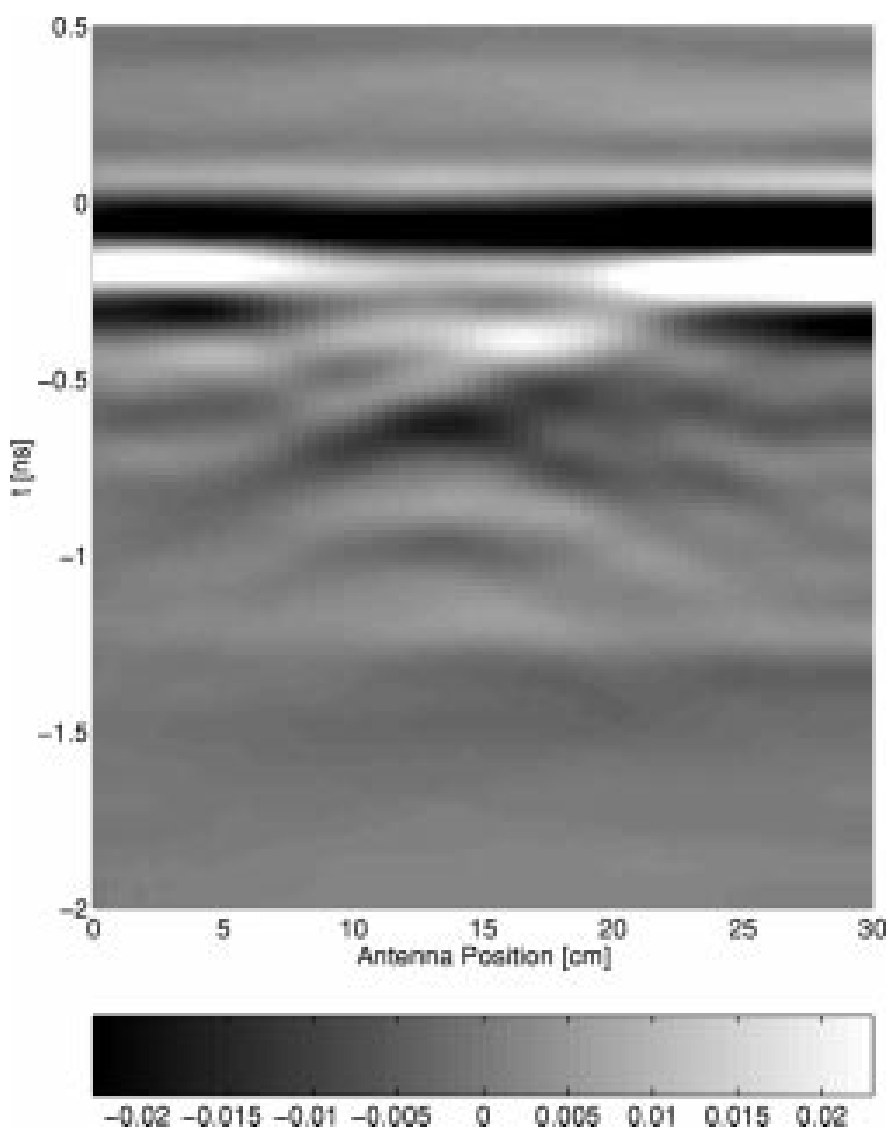

Fig. 10. Amplitude of the impulse response (for $1.2-6 \mathrm{GHz}$ data) for the PMA-3 mine plotted as functions of antenna positions (cm) and time (ns).

$\mathrm{GHz}$ band). This particular antenna operates for $1-6 \mathrm{GHz}$ frequency range, and it does not operate in the Rayleigh region of most of the A-P mines buried in clay. The PMA-3 mine was selected since the lower frequency limit of the radar system was just above the Rayleigh region of this target. The measured fields for this are shown in Fig. 11 for the region 1.2-6.0 $\mathrm{GHz}$. Note that the lowest frequency $(1.2 \mathrm{GHz})$ is just above the Rayleigh region. The data in Fig. 11 were supplemented as will be discussed by computed values for $0.1-1.2 \mathrm{GHz}$. To supplement these data in the Rayleigh region and remove this deficiency, the PMA-3 mine was approximated by a homogeneous dielectric body of the same overall shape, embedded in a lossless dielectric medium having relative permittivity the same as that of the clay ( $\epsilon_{x}^{\text {rel }} \approx 4$ for the observational bandwidth). The backscattered fields were then computed using an MoM solution for $0.1-1.2 \mathrm{GHz}$ range. The computed field data, after appropriate amplitude scaling and phase shifting, was cascaded with the measured data. The spectrum of PMA-3 $(0.1-6 \mathrm{GHz}$ frequency band), after supplementing the measured data with the numerical data, is shown in Fig. $11.1 /(j \omega)^{2}$ weighting is applied on this composite response, and the ramp response is finally obtained using IFT on this weighted frequency response. It is observed that the curve below $1.2 \mathrm{GHz}$ in Fig. 10 simply behaves as would be expected in the Rayleigh region and could have been estimated from the region of the curve above $1.2 \mathrm{GHz}$.

In practice, there are still several steps that would be required to create an image of the buried target. First, it would be neces-

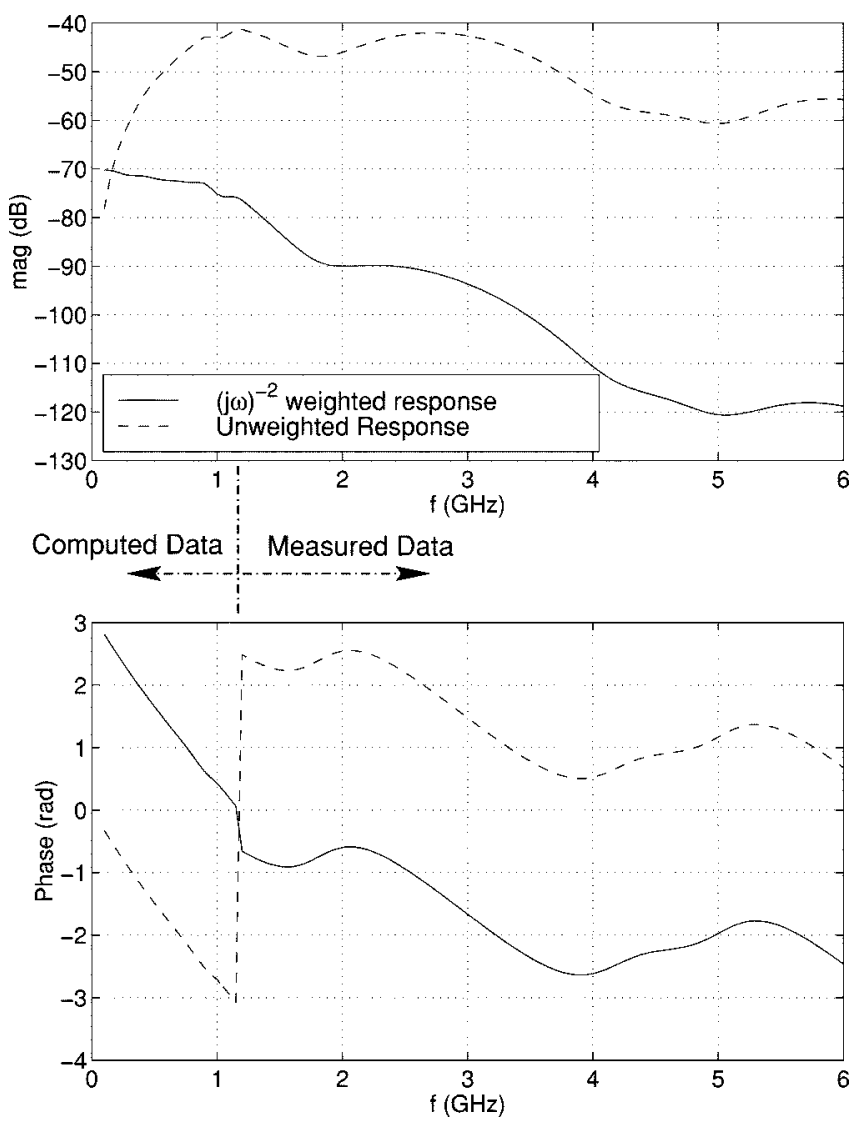

Fig. 11. The unweighted and $1 /(j \omega)^{2}$ weighted spectrum for the buried PMA-3 A-P mine, shown with the numerical data supplemented for frequencies below $1.2 \mathrm{GHz}$ with the measured data.

sary to demonstrate that an unknown target has rotational symmetry. This could be be achieved by moving the GPR in different directions across the center of the target and repeating the measurements of Fig. 10 to demonstrate that the target response does not change significantly as a function of position. Next, it is necessary to determine the lateral extent of the target. This can be achieved also using Fig. 10. It is visually estimated to be about $11.4 \mathrm{~cm}$. However, because the dielectric rod antenna is essentially a point source, the target is illuminated by the antenna even after it is no longer directly over the target. Since a target may not have a flat upper surface, there will be geometrically reflected rays from this surface, even though the antenna is not directly over the target. This results in an estimation of a bigger lateral extent of the target from a plot like Fig. 10. It is noted that a diameter, much closer to that of an actual target, has been obtained for a target with a flat upper surface.

Alternatively, the maximum transverse cross-sectional area $A_{\max }$ can also be estimated from the ramp waveform. This requires the scaling of the ramp response by a factor $\left[T_{12}(0) T_{21}(0) R_{23}(0)\right]^{-1}$, where $T_{12}(0)$ is the transmission coefficient of the wave at the free-space-ground interface for normal incidence, $T_{21}(0)$ is the transmission coefficient of the wave at the ground-free space interface for normal incidence, and $R_{23}(0)$ is the reflection coefficient of the wave (in the clay medium) illuminating the target at normal incidence. $R_{23}$ is assumed constant over the lit surface of the target. The lateral extent $l_{e}$ of the target, assuming that it is 


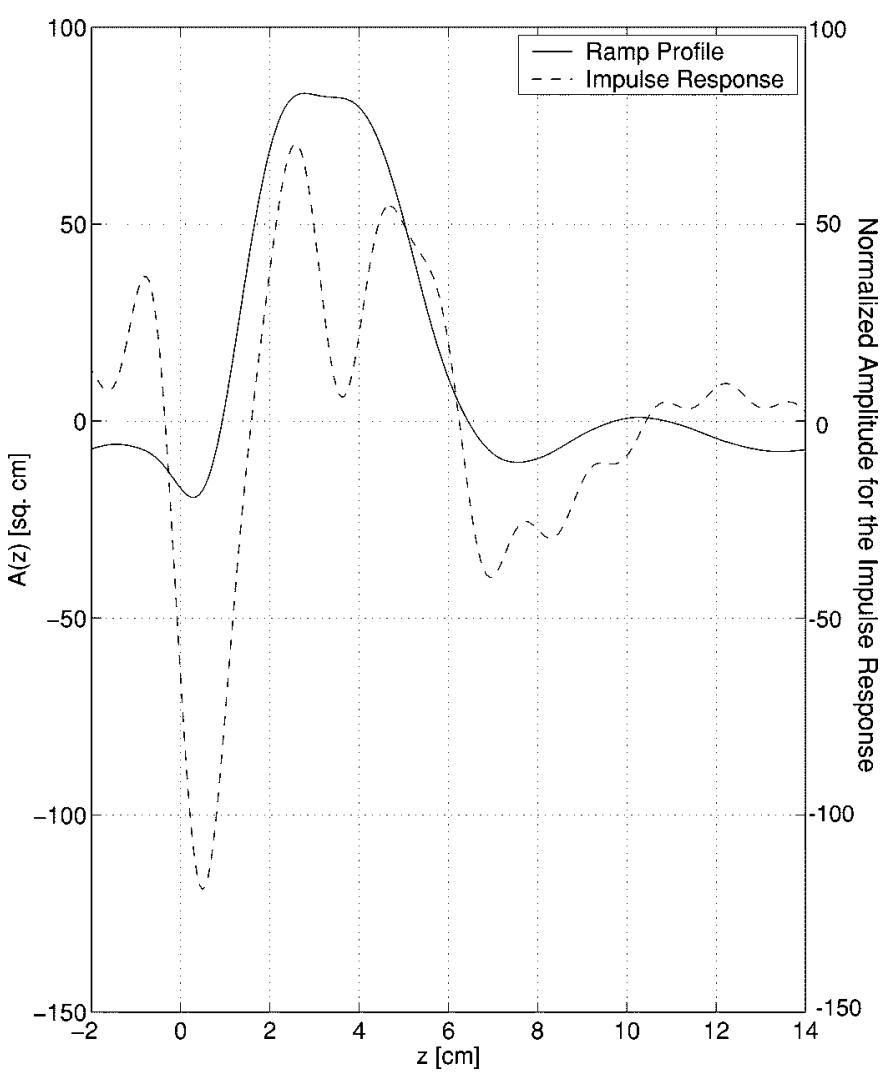

Fig. 12. The ramp profile function and the normalized impulse response obtained after supplementing $1.2-6 \mathrm{GHz}$ measured data withthe $0.1-1.1 \mathrm{GHz}$ MoM data.

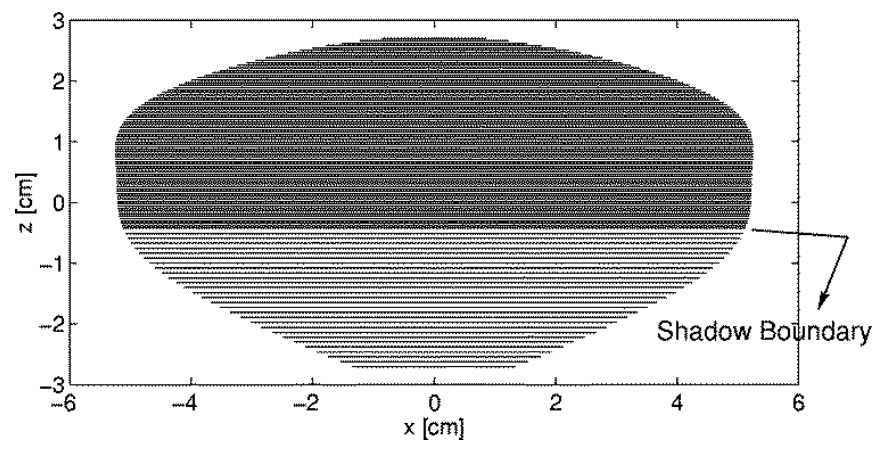

Fig. 13. Side-view image of PMA-3 A-P mine generated using the ramp profile function in Fig. 12.

rotationally symmetric, is obtained simply using the relation $l_{e}=2 \sqrt{A_{\max } / \pi}$. This process of estimation of $l_{e}$ of a buried land mine is reasonably good provided a) the incident wave entirely illuminates the target and b) the dielectric constant of the target is known. If a given land mine, embedded in a given ambient medium, is to be detected, its dielectric constant can be determined from the measured ramp waveform of that mine buried in the same ground condition. For most dielectric mines, reasonable values of the relative permittivity lie in the range 3-4 for our observational frequency band. For this particular case, the relative permittivities of free-space, clay, and the mine are, respectively, $\epsilon_{\text {air }}=1, \epsilon_{\text {clay }}=4$ and $\epsilon_{\text {mine }}=3 . A(z)$, obtained from the ramp response, and the normalized impulse response are shown in Fig. 12. The diameter of the PMA-3 mine, estimated from the ramp profile function, is $10.4 \mathrm{~cm}$, which is in good agreement with the actual diameter $10.3 \mathrm{~cm}$. Its image is shown in Fig. 13.

\section{CONCLUSION}

Transient scattered fields obtained from convex targets illuminated by a ramp incident waveform have been shown to provide a reasonable representation of the transverse physical cross section (or profile function) of the penetrable target as a function of range to the shadow boundary. However, a much less accurate representation is obtained beyond the shadow boundary. But even then, it is still a useful representation.

Ramp responses for several targets have been given, and it is shown that they do give good agreement with the target profile function. These cases include some land mines. Images have been generated for these targets, of different sizes and shapes, in free-space using the ramp response data from three orthogonal incidence angles.

A means of generating these orthogonal incidence angles for a shallow buried target is suggested that would make use of a modified version of the current dielectric rod antenna. However, this requires a rather cumbersome measurement. Therefore, a means of generating ramp images of a buried target is suggested using the broadside look angle ramp profile function, provided the shape of the target transverse to the direction of propagation is known. The approximate transverse shape of the target can be determined by observing the target in rotated planes about the center of the unknown response.

Preliminary approximate images have been generated for rotationally symmetric targets, such as a PMA-3 AP mine buried in dry clay at a JUXOCO site. The maximum value of the ramp profile function, obtained from the measured data, yields the dimension of the target in the transverse direction. Again, even in this cluttered medium, a realistic image of the target has been generated.

\section{REFERENCES}

[1] E. M. Kennaugh and D. L. Moffatt, "Transient and impulse response approximations," Proc. IEEE, pp. 893-901, Aug. 1965.

[2] J. D. Young, "Target imaging from multiple frequency radar returns," Ph.D. dissertation, The Ohio State University, Columbus, June 1971

[3] J. D. Young, K. A. Shubert, and D. L. Moffatt, "Synthetic radar imagery," IEEE Trans. Antennas Propagat., vol. AP-24, May 1976.

[4] S. Nag and L. Peters Jr., "Ramp response signatures for dielectric targets," in Proc. Soc. Photo-Opt. Instrument. Eng. Conf., Orlando, FL, Apr. 1998.

[5] C.-C. Chen, K. R. Rao, and R. Lee, "A new ultra-wide bandwidth dielectric rod antenna for ground-penetrating-radar applications," IEEE Trans. Antennas Propagat., to be published.

[6] D. L. Moffatt, "A physical optics approximation of the scattering for axial incidence from rotationally symmetric targets," Contract AF 30(602)-2042, Antenna Lab., The Ohio State Univ. Res. Foundation, Columbus, Rep. 925-6, Feb. 1961.

[7] S. Nag, "Ramp response signatures of dielectric scatterers," Ph.D. dissertation, The Ohio State University, 1999.

[8] S. Nag, L. Peters Jr., I. J. Gupta, and C.-C. Chen, "Ramp response signatures for the detection of anti-personnel mines," in Proc. Conf. Soc. Photo-Opt. Instrument. Eng., Orlando, FL, Apr. 1999.

[9] C.-C. Chen, "Design and development of enhanced ground penetrating radar systems for the detection and classification of unexploded ordnances and land mines," Ph. D. dissertation, The Ohio State Univ., Columbus, 1997

[10] E. H. Newman, "A User's Manual for the Electromagnetic Surface Patch Code: Preliminary Version ESP5.0," unpublished, June 1998. 
[11] C.-C. Chen, S. Nag, W. D. Burnside, J. Halman, K. Shubert, and L. Peters Jr., "A stand-off, focussed-beam land mine radar," IEEE Trans. GeoSci. Remote Sensing, to be published.

[12] A. van der Merwe, I. J. Gupta, and L. Peters Jr., "A clutter reduction technique for GPR data from mine like targets," in Proc. Conf. Soc. Photo-Opt. Instrument. Eng., Orlando, FL, Apr. 1999.

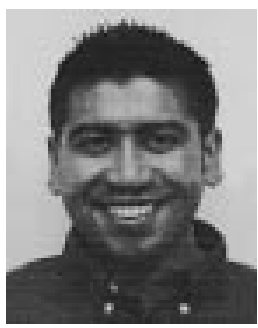

Soumya Nag (M'00) was born in Calcutta, India, in 1969. He received the B.E.E. degree from Jadavpur University, Calcutta, in 1991, the M.Eng. degree from Memorial University of Newfoundland, St. John's, Canada, in 1994, and the Ph.D. degree from The Ohio State University (OSU), Columbus, in 1999, all in electrical engineering.

From 1994 through 1999, he was a Graduate Research Associate with the ElectroScience Laboratory, OSU. He is currently a Signal Processing Engineer with Time Domain Corporation, Huntsville, AL. His research interests are in the fields of ultrawide-band radar, signal processing, electromagnetic scattering, and target identification.

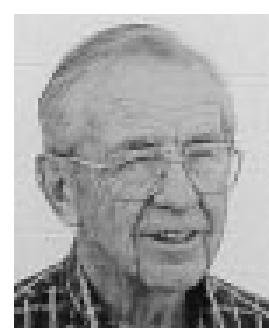

Leon Peters, Jr. (S'50-A'51-SM'60-F'81-LF'89) was born in Columbus, $\mathrm{OH}$. He received the B.E.E., M.Sc., and Ph.D. degrees from The Ohio State University (OSU), Columbus, in 1950, 1954, and 1959, respectively.

In 1950, he became a Research Associate in the OSU ElectroScience Laboratory (ESL) (formerly Antenna Laboratory), Department of Electrical Engineering. In 1960, he joined the teaching staff at OSU and has served as Technical Area Director for electromagnetic and remote sensing and as the Associate Department Chairman for research. From 1983 to 1994, he was the Director of the ESL. He is now a Professor Emeritus. His primary interest is now focused on ground-penetrating radar.

Dr. Peters is a member of Sigma Xi and Commission B of the International Union of Radio Science. He received the Distinguished Achievement Award from the Antenna Measurement Techniques Association in 1998. 